Article

\title{
Analysis of Lipids in the Medulla of Japanese Hair and Their Function
}

\author{
Jun Ymazaki ${ }^{1}$ and Kazuhisa Maeda ${ }^{1,2, * \text { (D) }}$ \\ 1 Bionics Program, Tokyo University of Technology Graduate School, Tokyo 192-0982, Japan; \\ g111604050@edu.teu.ac.jp \\ 2 School of Bioscience and Biotechnology, Tokyo University of Technology, Tokyo 192-0982, Japan \\ * Correspondence: kmaeda@stf.teu.ac.jp; Tel.: +81-42-6372442
}

Received: 18 March 2018; Accepted: 12 April 2018; Published: 15 April 2018

\begin{abstract}
The hair is composed of the cuticle (the outermost surface), cortex (its major part), and medulla (in the hair center). The lipid content of the medulla of Caucasian hair is relatively higher than that of African-American hair. However, the types of lipids therein remain unidentified. The aim of the current study was to analyze the constituent lipids of the medulla of Japanese hair, and to identify their function. A lipid peroxidase fluorescent reagent was used to investigate region-specific differences in the lipid content of the medulla (the tip, middle, and root portions). Since the medulla is important for the hair's glossiness, we also investigated the relationship between the lipid content and hair glossiness. The lipid content of hair, based on the absence or presence of lipid species in the medulla, was investigated using thin-layer chromatography (TLC). Micro-attenuated total reflectance Fourier-transform infrared spectrophotometry (micro-ATR FTIR) was used to analyze the similarities between the standard and medulla lipids, focusing on the methylene/methyl stretching vibration region. The data indicated that the medulla contained unsaturated lipids, the content of which decreased from the root to the tip of the hair. Hair glossiness was reduced with the decreasing lipid content, suggesting that unsaturated lipids of the medulla play a role in glossiness. The TLC analysis revealed differences in the type and amount of hair lipids in the medulla. While squalene and oleic acid spots were detected in hair with a continuously maintained medulla, these compounds were not detected in hair in which the medulla was not maintained. The medulla constituents similar to oleic acid and squalene were also identified by the micro-ATR FTIR spectrum analysis and the previous report. The findings indicate that the medulla is composed of at least squalene and oleic acid.
\end{abstract}

Keywords: medulla; lipids; micro-ATR FTIR; hair glossiness

\section{Introduction}

Hair is largely composed of the cuticle, i.e., its outermost surface; the cortex, the main body of the hair; and the medulla, the core of the hair [1]. The medulla is largely composed of non-keratinized proteins, rendering it relatively soft, and contains many cavities, much like a sponge. As a result of the multiple small air pockets inside, it is easily cracked when exposed to external stimuli. When these small air chambers are vacuolated, small intercellular cracks remain during keratinization, separated by a partition wall. Considerable quantities of air are captured in this manner, and the process is thought to aid heat-retention, with the air acting as insulation. For this reason, there is a tendency for the medulla to proportionally increase in animals living in relatively cold regions [2,3].

By contrast, in human-in comparison with other animals-the proportionate amount of the medulla is as small as $5 \%$ or lower. Although there are instances when the hair grows out hollow from the root, in many cases, the heat of hairdryers and the stretching force generated by brushing can easily cause the hollowing [4]. Moreover, the cavities inside the cortex render the entire fiber 
cloudy. When the hollowing occurs in the medulla, a white streaky line is prominently and clearly observed in the central portion of the hair, and hair glossiness is reduced [5]. It is believed that this is an outcome of a diffuse reflection of light penetrating the cavity, which causes the hair to take on a whitish tone and appear faded [5]. The medulla of mammalian hair is present in the core of some hair shafts, but by no means in all hair [6]. The medulla may not be present, may be broken, or, else, may be continuous [6-10]. At the terminal differentiation stage of the hair, the cells of the medulla create larger cavities. One explanation for this phenomenon is that these cavities are filled with air and provide an insulating quality. In contrast to other parts of the hair follicle, the morphological appearance of the medulla is significantly different across the animal kingdom. In many mammals, the distinct spaces are arranged in a continuous ladder-shape-arranged in a regular, alternating pattern, and the structure of the mature medulla is highly regular. By contrast, in other mammals, including humans-the medulla has a singular or otherwise multiple non-systematic structure consisting of highly interstitial cells $[6,9,11]$. Electron microscopy and X-ray analysis [11-13], as well as cysteine analysis of the medulla of animal hair $[14,15]$, indicate that $\alpha$-keratins are present in the medulla [16-18]. However, the medulla is poor in cystine and rich in citrulline [3]. The total amount of lipid that is extractable from hair is generally $1-9 \%$ of the weight of the hair [1]. The lipids of hair were analyzed as $58.1 \%$ fatty acids (covalent and non-covalent), 19.8\% wax ester, $9.7 \%$ hydrocarbon, $5.2 \%$ cholesterol, $2.8 \%$ squalene, and others [19-21]. Approximately $51 \%$ of the non-covalently bound fatty acids was unsaturated fatty acid (primarily palmitoleic acid and oleic acid) in human hair [22]. A Raman imaging method was developed to visualize the distribution of squalene in Chinese white virgin hair from the cuticle to the medulla [23]. Major constituents of the integral hair lipids are, in decreasing order, fatty acids, phytosphingosine, and ceramide [24]. The medulla, a porous structure with vacuoles or granules, is made up by an unidentified material $[15,25]$. The medulla contains more lipids than the rest of the hair fiber [26], but their composition is unclear. The medulla of Caucasian hair has more lipids than that of African-American hair. Further, the cuticles of both Caucasian and African-American hair contain very few lipids [26]. As the medulla is not present in fine or soft hair, it is believed that the human hair medulla is not indispensable to the composition of hair; its role is yet to be fully understood. Moreover, cosmetics research indicates that the medulla impacts the external appearance of hair [5].

The purpose of the current study was to analyze the lipid component of the medulla and its relationship with hair glossiness.

\section{Materials and Methods}

\subsection{Materials}

Liperfluo was purchased from Dojindo Laboratories (Kumamoto, Japan). Dimethyl sulfoxide (DMSO), acetone, oleic acid, linoleic acid, triolein, methyl oleate, cholesterol oleate, methanol, chloroform, hexane, benzene, diethyl ether, acetic acid, and copper sulfate (II) solution were obtained from Wako Pure Chemical Industries, Ltd. (Osaka, Japan). $\alpha$-Linolenic acid was purchased from Nacalai Tesque, Inc. (Kyoto, Japan). Squalene was from Sigma-Aldrich Corp. (St. Louis, MO, USA). Tissue-Tek Cryomold no. 3 and Tissue-Tek O.C.T. compound were purchased from Sakura Finetek Japan, Ltd. (Tokyo, Japan). Aluminum thin-layer chromatography (TLC) plates coated with silica gel 60 F254 produced by Merck \& Co., Inc. (Kenilworth, NJ, USA) were used. IR-7090 KBr-REAL crystal IR card and IR-7093 KBr-REAL cover slips were purchased from System Engineering, Inc. (Tokyo, Japan). Capcell Pak C18 UG120 column ( $4.6 \times 250$ mm² , C18 column; Shiseido Co., Ltd.; Tokyo, Japan) was used for high-performance liquid chromatography (HPLC).

\subsection{Equipment}

Lightning Cure LC6 (150 W xenon lamp L8253; Hamamatsu Photonics K. K.; Hamamatsu, Japan) lamps were used as the UV source. A long-pass filter with a $280 \mathrm{~nm}$ cutoff (A9616-03; Hamamatsu Photonics K. K.; Shizuoka, Japan) was used in these lamps [27,28]. Hair glossiness was examined using 
a Glossymeter GL20 (Courage+Khazaka Electronic GmbH; Köln, Germany). Micro-attenuated total reflectance Fourier-transform infrared spectrophotometry (micro-ATR FTIR) was performed using FTS-6000 and UMA-500 (Digilab Japan, Tokyo, Japan).

\subsection{Hair Samples}

Hairs cut from Japanese individuals were used in the current study. The hair samples had never been exposed to chemical treatment, such as perming, dyeing, and/or bleaching. The samples were sealed in zip-lock bags and stored in a dark place.

\subsection{Determination of the Unsaturated Lipid Content of the Medulla}

The hairs were cut into three $2-\mathrm{cm}$ long sections, containing the tip, middle, and root $(n=3)$. The base of the Cryomold no. 3 was filled with the O.C.T. compound (an embedding agent for preparing frozen tissue samples) and placed over dry ice, and the prepared hair samples were inserted vertically into the O.C.T. compound. Each hair sample was held in place with tweezers until the O.C.T. compound froze solid, at which point the frozen hair was prepared for analysis. Using a cryostat microtome, the hair samples were cut into $20-\mu \mathrm{m}$ slices, and transferred to glass slides. Three parts for each section were prepared (nine in total: three from the tip, three from the middle, and three from the root) and were kept at $-30{ }^{\circ} \mathrm{C}$ before further analysis. Liperfluo was used to determine the unsaturated lipid content of the hair samples. Liperfluo is a triphenylphosphine-moiety reagent, which specifically reacts with unsaturated lipids, its perylene rings giving off a strong fluorescence. Liperfluo ( $1 \mathrm{mmol} / \mathrm{L}$ in DMSO) was diluted in pure water to $2 \mu \mathrm{mol} / \mathrm{L}$. Then, $10 \mu \mathrm{L}$ of the dilution was dropped onto each hair slice sample and incubated at $37^{\circ} \mathrm{C}$ for $30 \mathrm{~min}$. Sample fluorescence was observed using an Olympus BX51 fluorescence microscope (Olympus Corporation; Tokyo, Japan) equipped with a microscope digital camera (Olympus BP71). In addition, the cross-sectional diameter of each hair was measured. The fluorescence intensity of unsaturated lipids in the hair medulla was quantified using the Image J image analysis software.

\subsection{Determination of the Unsaturated Lipid Content in the Medulla of Hair with Lipid Content Reduced by Acetone or Chloroform Treatment}

Acetone or chloroform was added to a 100-mL beaker. Then, glass slides containing cut hair sections were placed vertically in the beaker and heated overnight at room temperature. Following this, $10 \mu \mathrm{L}$ of Liperfluo $(2 \mu \mathrm{mol} / \mathrm{L})$ was added, using an eyedropper to each dried slice of hair and the samples were incubated at $37^{\circ} \mathrm{C}$ for $30 \mathrm{~min}$. Sample fluorescence was then observed using a fluorescence microscope. The cross-sectional diameter of the hair was also measured. Image analysis software, Image J (National Institutes of Health, Bethesda, MD, USA), was used to quantify the fluorescence intensity of unsaturated lipids in the hair medulla.

\subsection{Changes in the Glossiness of Hair with Reduced Lipid Content in the Medulla}

Black hair bundles were placed in a screw-cap beaker to which acetone was added, and then heated overnight at room temperature to reduce the cuticle lipid content. The samples were then treated with acetone or chloroform. The acetone or chloroform treatment involved adding acetone or chloroform to a 100-mL beaker, inserting the hair bundle, and leaving it to stand overnight at room temperature. The glossiness of the untreated group (control group) and the group treated with acetone or chloroform was examined, using the Glossymeter GL20, immediately after removing the solvent and drying. To obtain a gloss value that was mostly independent of the hair color, the diffuse light entering the gloss channel was subjected to a diffuse scattering correction (DSC).

\subsection{Analysis of Hair Lipids Using TLC}

Using a transmission microscope, hairs were sorted into those with and without the medulla. For the TLC analysis, the developing solvents were hexane, benzene, chloroform, methanol, diethyl 
ether, acetic acid, and copper sulfate solutions. Oleic acid, linoleic acid, $\alpha$-linolenic acid, squalene, triolein, methyl oleate, and cholesterol oleate were used as standards. For the TLC analysis of hair lipids, the hair samples were placed in a screw-cap beaker containing acetone and left overnight at room temperature to reduce the lipid content of the cuticle. Following this, $500 \mu \mathrm{L}$ of the acetone hair sample was transferred to a $1.5-\mathrm{mL}$ test tube and dried on a heating block set to $60{ }^{\circ} \mathrm{C}$ in bubbling nitrogen gas. Then, $250 \mu \mathrm{L}$ of the lipid-extracting solvent was added (chloroform-methanol, 2:1), and agitated for $30 \mathrm{~s}$ in a vortex-mixer, following which the lipids were extracted. The samples were centrifuged for $1 \mathrm{~min}$ and $150 \mu \mathrm{L}$ of the lower layer was transferred to another micro-tube and dried. Following this, $4 \mu \mathrm{L}$ of the lipid-extracting solvent was added and the mixture was used as a sample for TLC analysis. The entire sample, together with $2 \mu \mathrm{L}$ of the standard solution, was then spotted onto a TLC plate. After drying, the plate was sequentially developed using developing solutions $\mathrm{A}$ to $\mathrm{D}$ (solution A, $10 \mathrm{~mL}$ hexane; solution B, $10 \mathrm{~mL}$ benzene; solution $\mathrm{C}, 9.5 \mathrm{~mL}$ chloroform and $2 \mathrm{~mL}$ methanol; solution D, $8 \mathrm{~mL}$ hexane, $2 \mathrm{~mL}$ diethyl ether, and $0.1 \mathrm{~mL}$ acetic acid) [28]. The plates were then colored by heating with $3 \%$ copper (II) sulfate and 15\% aqueous phosphoric acid solutions.

\subsection{Lipid Spectral Analysis Using Micro-ATR FTIR}

The micro-ATR FTIR evaluation was performed using Digilab Japan apparatus (FTS-6000, UMA-500). Frozen cross-sections of hair $(20 \mu \mathrm{m})$ were applied to a KBr-IR card and covered with KBr-REAL cover slips. The measurement was conducted by micro-attenuated total reflectance (microscopic ATR) at the following settings: scope of analysis, the medulla; measurement spectrum range, $3120-2800 \mathrm{~cm}^{-1}$; resolution, $8 \mathrm{~cm}^{-1}$; sequences, 64 . The micro-ATR FTIR spectra of samples and standards were analyzed by eight-point smoothing of the second derivative. Spectra comparisons were performed using pure oleic acid, linoleic acid, $\alpha$-linolenic acid, squalene, triolein, methyl oleate, and cholesterol oleate.

\subsection{Statistical Analysis}

The significance of measurement was determined using an unpaired t-test using Microsoft Excel statistics.

\section{Results}

\subsection{Determination of the Unsaturated Lipid Content of the Medulla}

Figure 1 displays the fluorescence intensity of unsaturated lipids observed using a fluorescence microscope. The size of the medulla, as well as the fluorescence intensity associated with unsaturated lipids, differed depending on the observed part of the hair samples.
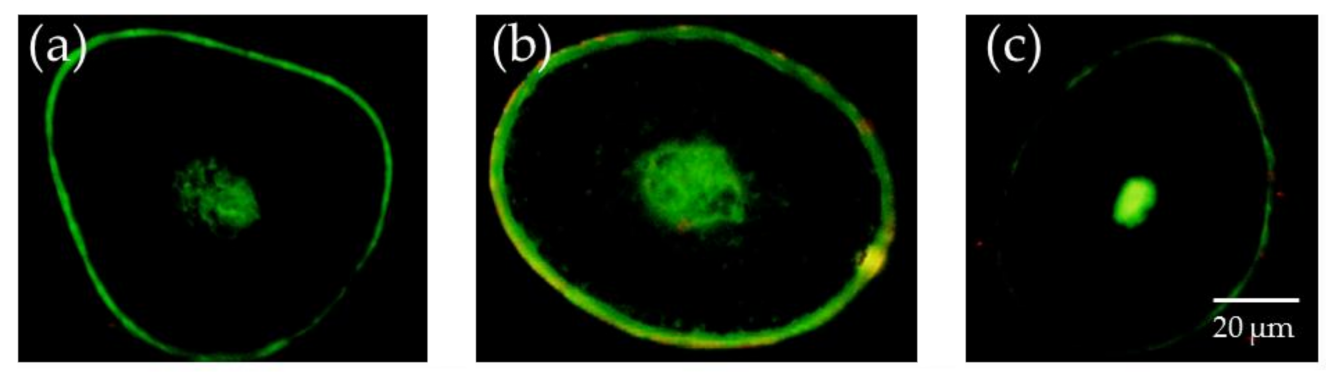

Figure 1. Unsaturated lipid content of each cross-section of hair (200× magnification). (a) Hair tip; (b) middle of the hair; and (c) hair root.

Moving from the hair root to the tip, the intensity of fluorescence associated with unsaturated lipids in the medulla decreased. Unsaturated lipids were also observed in the cuticle. Figure 2 presents the observed fluorescence intensity of lipids in the medulla, created using the Image J analysis software. 
As displayed in Figure 2, a comparison of the relative fluorescence intensity of the medulla revealed decreasing fluorescence from the root to the tip, and that the root contained more unsaturated lipids than the tip.

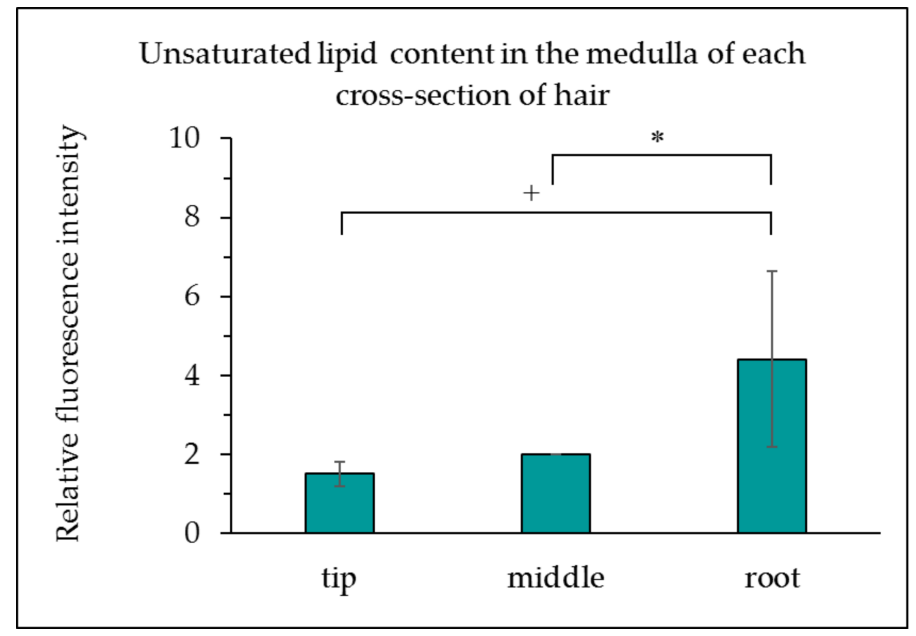

Figure 2. Unsaturated lipid content in the medulla of each cross-section of hair. The results are expressed as the mean \pm standard deviation from three experiments. $* p<0.05,^{+} p<0.1$.

\subsection{Determination of the Unsaturated Lipid Content in Hair with Lipid Content Reduced by Acetone and Chloroform Treatment}

Figure 3 displays the intensity of fluorescence from unsaturated lipids observed using a fluorescence microscope. The fluorescence of unsaturated lipids in hair samples treated with acetone or chloroform was lower than that of the untreated control. In contrast, the fluorescence intensity of unsaturated lipids in the cuticle remained unchanged. Figure 4 displays the relative fluorescence intensity of lipids in the medulla. The unsaturated lipid content in the medulla was higher than that in the cuticle (Figure 3), and almost all unsaturated lipids of the medulla were removed by acetone and chloroform. The bunch of hair was consequently dipped in those solvents to deplete the unsaturated lipids from the cuticle.

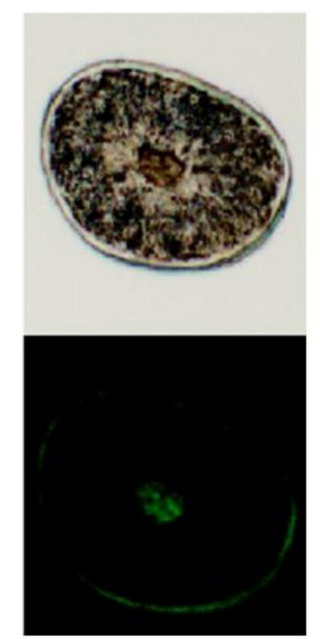

control

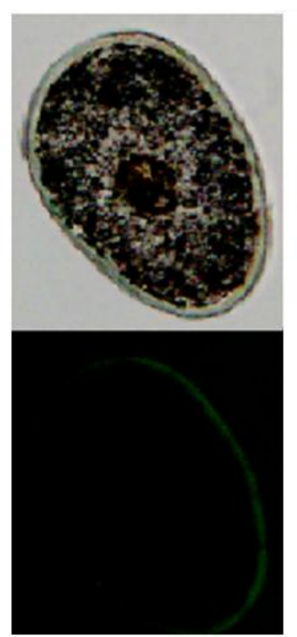

acetone

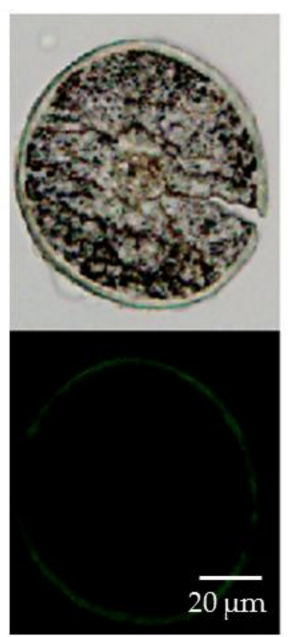

chloroform

Figure 3. Fluorescence of unsaturated lipids in hair treated with acetone or chloroform $(200 \times$ magnification). 
As indicated in Figure 4, comparing the fluorescence intensity of the unsaturated lipids in the treated medulla, the fluorescence of unsaturated lipids in the medulla of hair slices treated with acetone or chloroform was greatly decreased. Based on this observation, it was concluded that the medulla does indeed contain unsaturated lipids.

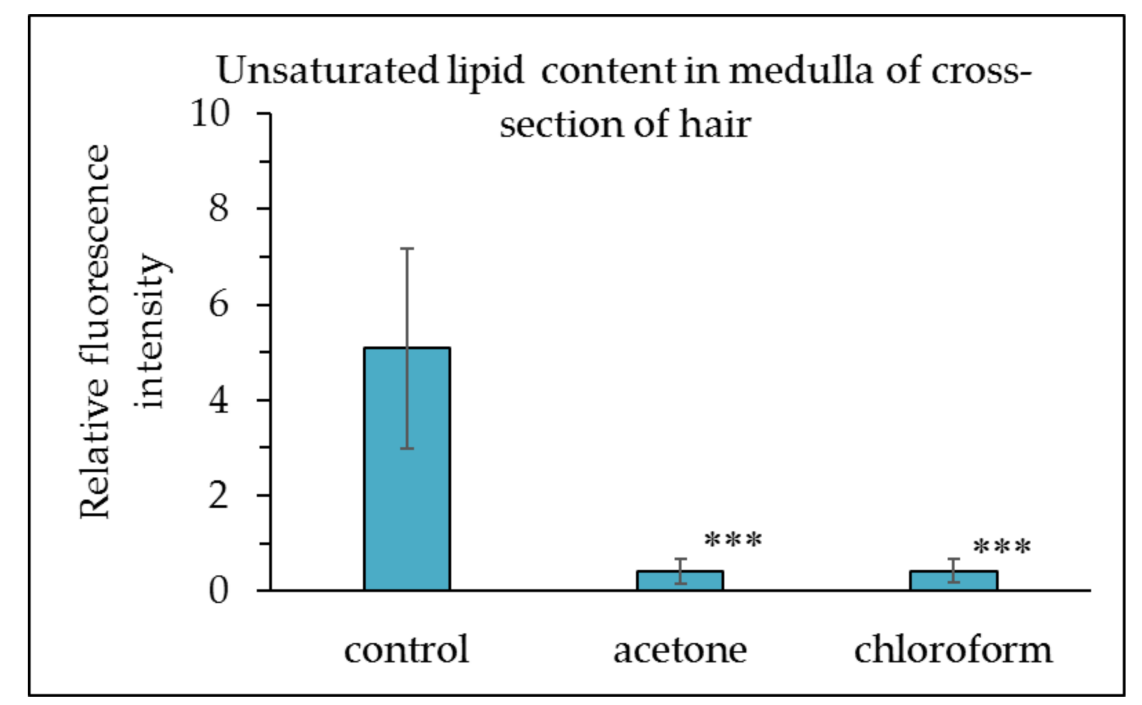

Figure 4. Unsaturated lipid content in medulla of the hair cross-section following lipid content reduction by an acetone or chloroform treatment. The results are expressed as the mean \pm standard deviation from 10 experiments. ${ }^{* * *} p<0.001$ vs. control.

\subsection{Changes in the Glossiness of Hair with the Lipids Removed from the Medulla}

As indicated in Figure 5, the glossiness of the hair bundles treated with acetone or chloroform was dramatically reduced. The acetone treatment reduced the glossiness of the hair to a greater extent than the chloroform treatment.

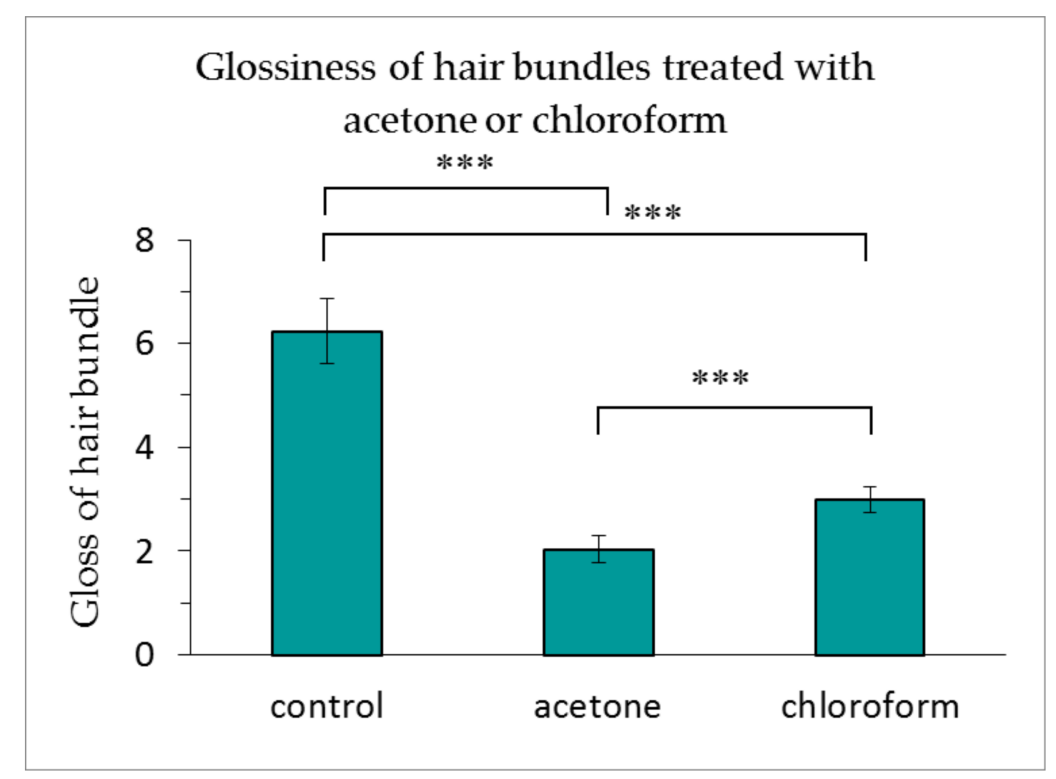

Figure 5. Glossiness of hair bundles treated with acetone or chloroform. The results are expressed as the mean \pm standard deviation from 10 experiments. ${ }^{* * *} p<0.001$. 


\subsection{Hair Lipid Analysis Using TLC}

Figure 6 presents the results of the TLC analysis of hairs with and without the medulla. The TLC analysis revealed that hairs with the medulla contained a greater variety of lipids than those without the medulla (Figure 6a). Moreover, hairs with the medulla-with dense lipid spots-were found to have more lipids than those without the medulla (Figure 6a). Furthermore, based on the Rf (relative to front) values of the lipid standards, oleic acid and squalene were present in hair with the medulla; squalene was not present in hair without the medulla (Figure 6b).

(a)
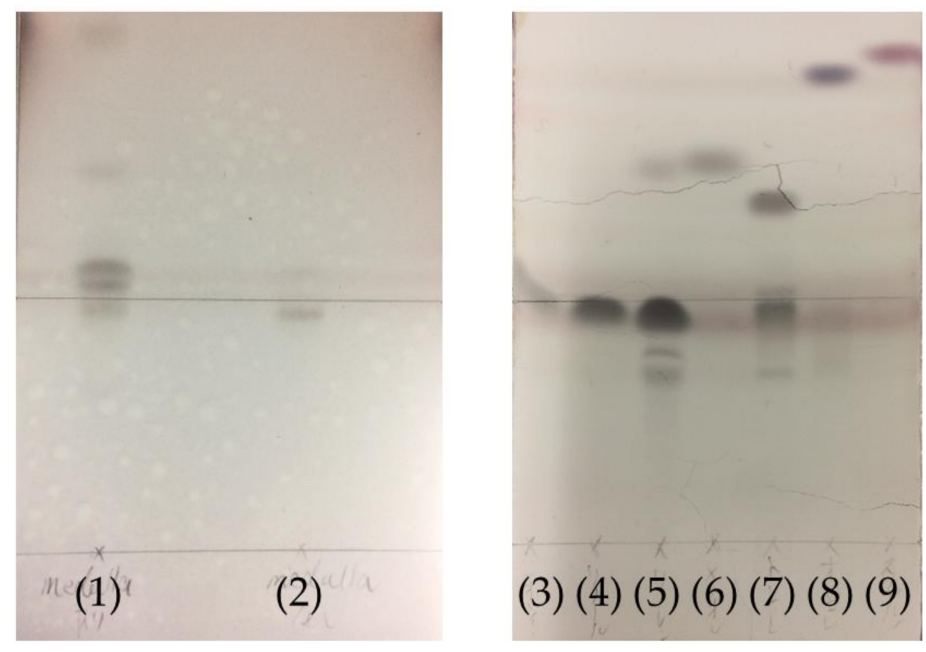

(b)

\begin{tabular}{|c|c|}
\hline lipids & Rf value \\
\hline oleic acid & 0.46 \\
\hline linoleic acid & 0.43 \\
\hline$\alpha$-linolenic acid & 0.43 \\
\hline squalene & 0.72 \\
\hline triolein & 0.63 \\
\hline methyl oleate & 0.87 \\
\hline $\begin{array}{c}\text { cholesterol } \\
\text { oleate }\end{array}$ & 0.91 \\
\hline
\end{tabular}

\begin{tabular}{|c|c|c|}
\hline hairs & Rf value & \\
\hline \multirow{3}{*}{ with medulla } & 0.98 & unknown \\
\cline { 2 - 3 } & 0.72 & squalene \\
\cline { 2 - 3 } & 0.54 & unknown \\
\cline { 2 - 3 } & 0.51 & unknown \\
\cline { 2 - 3 } & 0.46 & oleic acid \\
\hline \hline without medulla & 0.46 & oleic acid \\
\hline
\end{tabular}

Figure 6. (a) Lipid analysis of hair with and without the medulla using thin-layer chromatography (TLC). Designations in Figure 6a: (1) hairs with the medulla; (2) hairs without the medulla; (3) oleic acid; (4) linoleic acid; (5) $\alpha$-linolenic acid; (6) squalene; (7) triolein; (8) methyl oleate; and (9) cholesterol oleate. (b) Rf (relative to front) values for lipid standards and lipids obtained by lipid extraction from hair with and without the medulla, as described in the Section 2.7.

\subsection{Spectral Analysis of Lipids Using Second Derivative Micro-ATR FTIR}

The second derivative micro-ATR FTIR spectrum $\left(3120-2800 \mathrm{~cm}^{-1}\right)$ of each sample is displayed in Figure 7. A comparison of the respective spectra of the medulla and lipid standards is presented, confirming the similarity of the spectrum of medulla with the spectrum of squalene. The distinctive spectra of oleic acid, linoleic acid, $\alpha$-linolenic acid, triolein, methyl oleate, and cholesterol oleate could not be confirmed. 

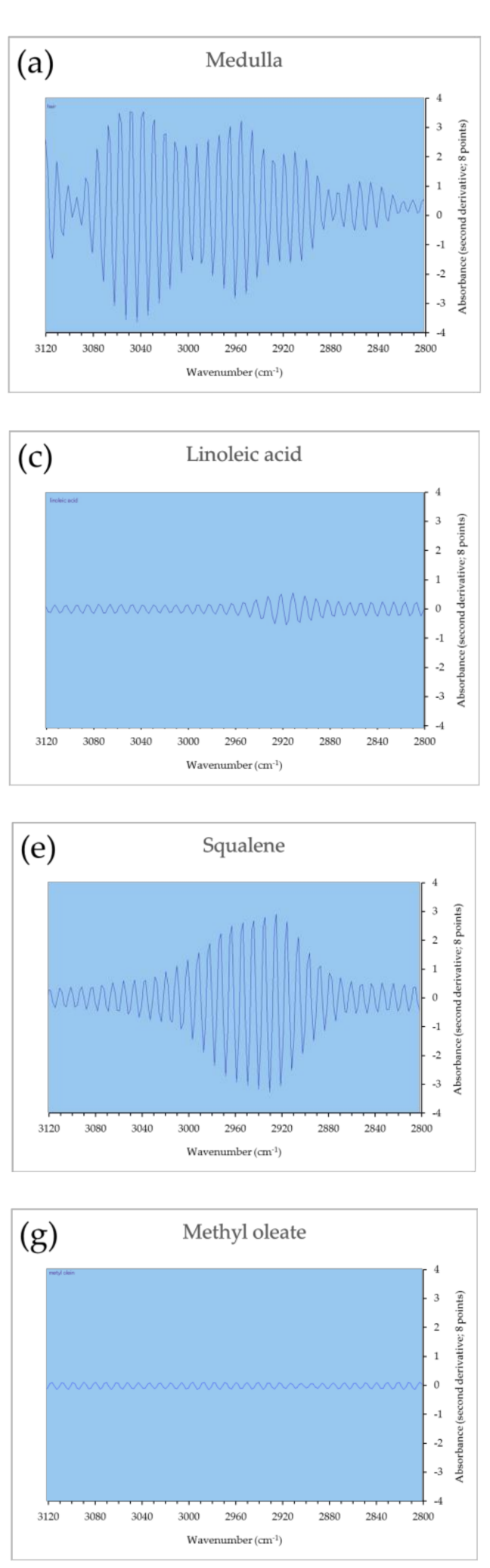
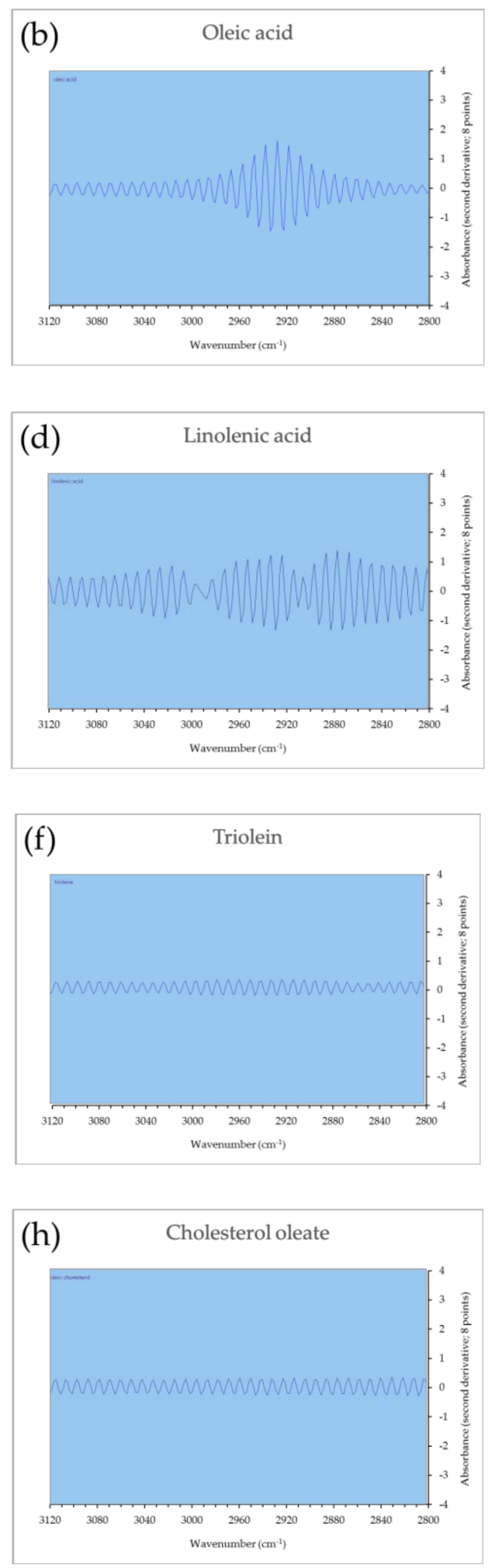

Figure 7. Second derivative micro-attenuated total reflectance Fourier-transform infrared spectrophotometry (micro-ATR FTIR) spectra of the medulla and lipids. (a) Medulla, (b) oleic acid, (c) linoleic acid, (d) $\alpha$-linolenic acid, (e) squalene, (f) triolein, (g) methyl oleate, and (h) cholesterol oleate. 


\subsection{Spectral Analysis of Micro-ATR FTIR}

The micro-ATR FTIR spectra (4000-800 $\mathrm{cm}^{-1}$ ) of medulla and oleic acid are displayed in Figure 8. A comparison of the respective spectra of the medulla and oleic acid standard is presented, confirming the similarity of the spectrum of medulla with the spectrum of oleic acid in the wavenumber range of $3100-2800 \mathrm{~cm}^{-1}$, which is derived from the methylene and methyl stretching frequencies. The distinctive spectra of $1720-1700 \mathrm{~cm}^{-1}$ could be observed, which is derived from CO stretching frequency in carboxylic acid dimer.

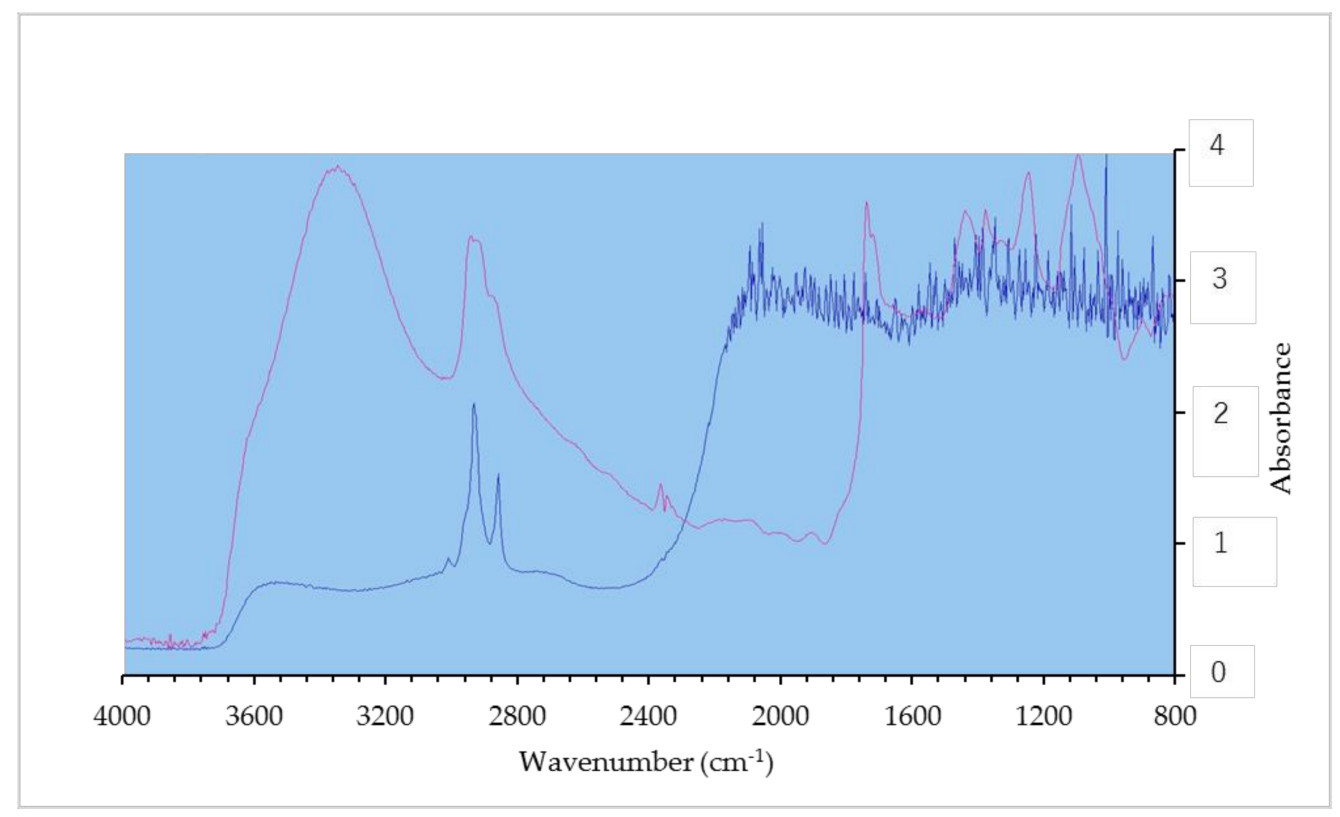

Figure 8. Micro-ATR FTIR spectra of the medulla and oleic acid. Red line-medulla and blue lineoleic acid.

\section{Discussion}

The current study revealed that the medulla contains unsaturated lipids. As the amount of lipids is lower at the hair tip and higher at the hair root, we propose that more unsaturated lipids are present in the medulla at the hair root than in that of the hair tip. Acetone or chloroform penetrated the interior of the hair and reduced the lipid content of the medulla, since the fluorescence intensity of frozen slices of hair that had been treated with either of these solvents was reduced.

The TLC analysis indicated changes in the quantity and quality of unsaturated lipids in hair, depending on the presence of the medulla. Hence, we propose that unsaturated lipids from the medulla penetrate the cortex and the cuticle, and ultimately, the hair surface. Since unsaturated lipids play a role as both nutrients and a barrier in the body, it is likely that the medulla-containing these lipids-is a source of unsaturated lipids for the hair.

In hairs in which the medulla is consistently present, much like a pencil lead, oleic acid and squalene were detected, while squalene was not detected in hairs without the medulla. Consequently, the second derivative micro-ATR FTIR analysis was performed to establish the type of lipids present in the medulla. The analysis revealed the presence of multiple lipids (at unknown ratios) in the medulla. Although the ratios could not be determined, second derivative micro-ATR FTIR was detected near the wave numbers $3040,2960,2920$, and $2850 \mathrm{~cm}^{-1}$, with different lipids contributing to the signals. The second derivative micro-ATR FTIR spectrum of squalene was detected from 3020 to $2860 \mathrm{~cm}^{-1}$, while the second derivative micro-ATR FTIR spectrum of oleic acid was detected from 2980 to $2860 \mathrm{~cm}^{-1}$. The infrared spectrum peak at $2955 \mathrm{~cm}^{-1}$ was generated by the $\mathrm{CH}_{3}$ asymmetric stretch; the peak at $2925 \mathrm{~cm}^{-1}$ was generated by the $\mathrm{CH}_{2}$ asymmetric stretch; and the peak at $2854 \mathrm{~cm}^{-1}$ 
was generated by the $\mathrm{CH}_{2}$ symmetric stretch [29]. The H-bonded carboxylic acid dimer was detected from 3300 to $2500 \mathrm{~cm}^{-1}$ [30]. The peak near the wavenumber $3300 \mathrm{~cm}^{-1}$ represented a hydrophilic functional group [30]. This indicated that oleic acid was present in the dimer in the medulla. It is thought that the peak at $3040 \mathrm{~cm}^{-1}$ signified quasi-double bonds, which originated from the double bonds, because the very small amount of sample was measured by the ATR method [31]. The methylene and methyl stretching frequencies, which appeared in the wavenumber range of $3100-2800 \mathrm{~cm}^{-1}$, are known to be associated with lipids [29,30,32]. The CO stretching frequency, which appears in the wavenumber range of $1720-1700 \mathrm{~cm}^{-1}$, is known to be associated with a carboxylic acid dimer $[29,30,32]$. They suggest that at least squalene and dimerized oleic acid were present in the medulla. Human sebum consists of triglycerides ( $30-50 \%)$, followed by wax esters ( $26-30 \%)$, fatty acids $(15-30 \%)$ and squalene (12-26\%) [33]. Both squalene and oleic acid in the medulla of hair are thought to be derived from the sebaceous gland. In addition, oleic acid forms a dimer in an aqueous solution [34] and it is thought to form condensed aggregates of lamellar bilayers at physiological $\mathrm{pH}[35,36]$. It was reported that unsaturated fatty acids were more abundant in the hair cortex than at the hair surface, and oxidative metabolites derived from linoleic acid and $\alpha$-linolenic acid were found in the hair bulb and melanin granules [37]. We propose that unsaturated lipids are supplied to the tip from the root, inside the cave of the medulla, and that the unsaturated lipid content decreases from the root to the tip since the unsaturated lipids in the medulla are liquid fluidity. Furthermore, lipid depletion from the medulla dramatically reduced hair fluorescence, suggesting that the unsaturated lipids present in the medulla are involved in hair glossiness. In hair with the medulla, in which the structure of the medulla is damaged and fine air bubbles form instead of lipids, the refractive index is reduced. The difference in the refractive index between the medulla with fine air bubbles and the cortex with keratin proteins increases, the amount of light penetrating the hair is reduced, and the amounts of both penetrating light and scattered light are reduced, negatively affecting hair glossiness. Unsaturated lipids are present not only in the medulla, but also in the cuticle edge. A possibility exists, therefore, that the acetone and chloroform treatment of hair also depleted lipids from the cuticle edges. Nevertheless, because the presence of unsaturated lipids in the cuticle is limited to the edge of the cuticle [28], it is believed that the relationship between unsaturated lipids and hair glossiness is primarily associated with the lipids present in the medulla.

\section{Conclusions}

It has been reported that lipids are present in the human hair medulla, but their composition was unclear. Here, we demonstrated that the medulla of sampled Japanese hair contained liquid unsaturated fatty acids and unsaturated hydrocarbons, comprised of at least squalene and oleic acid, which were most abundant at the root of the medulla, rather than the tip. Furthermore, unsaturated lipids may be linked to hair glossiness, since a remarkable decrease in glossiness was observed in the hairs from which the unsaturated lipids were depleted.

Author Contributions: Jun Yamazaki and Kazuhisa Maeda designed the study, and performed the experiment and the data analysis. Jun Yamazaki and Kazuhisa Maeda interpreted the data and drafted the manuscript. Kazuhisa Maeda supervised the study and critically revised the manuscript. All authors read and approved the final manuscript.

Conflicts of Interest: The authors declare no conflicts of interest.

\section{References}

1. Robins, C.R. Chemical and Physical Behavior of Human Hair, 5th ed.; Springer: Berlin/Heidelberg, Germany, 2012.

2. Nomura, Y. Doubutsumou No Keitaikansatsu. Hokkaido Univ. Collect. Sch. Acad. Pap. 1995, 2, 3-11. (In Japanese)

3. Clement, J.L.; Hagege, R.; Le Pareaux, A.; Carteaud, J.P. Ultrastructural study of the medulla of mammalian hairs. Scan. Electron Microsc. 1981, Pt 3, 377-382. 
4. Satoh, N. Mechanism of beautifulness of hair and the structure factors. J. Surf. Sci. Soc. Jpn. 2006, 27, 480-484. (In Japanese) [CrossRef]

5. Nagase, S.; Shibuichi, S.; Ando, K.; Kariya, E.; Satoh, N. Influence of internal structures of hair fiber on hair appearance. I. Light scattering from the porous structure of the medulla of human hair. J. Cosmet. Sci. 2002, 53, 89-100. [PubMed]

6. Morioka, K. Hair Follicle. Differentiation under the Electron Microscope. An Atlas; Academic Press: Tokyo, Japan, 2005; pp. 25-43.

7. Brown, F.M. The microscopy of mammalian hair for anthropologists. Proc. Am. Philosoph. Soc. 1942, 85, 250-274.

8. Hutchinson, P.E.; Thompson, J.R. The size and form of the medulla of human scalp hair is regulated by the hair cycle and cross-sectional size of the hair shaft. Br. J. Dermatol. 1999, 140, 438-445. [CrossRef] [PubMed]

9. Deedrick, D.W.; Koch, S.L. Microscopy of hair Part I: A practical guide and manual for human hairs. Forensic. Sci. Commun. 2004, 6, 1.

10. De Cássia Comis Wagner, R.; Kiyohara, P.K.; Silveira, M.; Joekes, I. Electron microscopic observations of human hair medulla. J. Microsc. 2007, 226, 54-63. [CrossRef] [PubMed]

11. Parakkal, P.F.; Matoltsy, A.G. A study of the differentiation products of the hair follicle cells with the electron microscope. J. Invest. Dermatol. 1964, 43, 23-34. [CrossRef]

12. Deedrick, D.W.; Koch, S.L. Microscopy of hair Part II: A practical guide and manual for animal hairs. Forensic. Sci. Commun. 2004, 6, 3.

13. Roth, S.I. Hair and nail. In Ultrastructure of Normal and Abnormal Skin; Zelickson, A.S., Ed.; Lea \& Febinger: Philadelphia, PA, USA, 1967; pp. 105-131.

14. Rogers, G.E.; Kuczek, E.S.; Mackinnon, P.J.; Presland, R.B.; Fietz, M.J. Special biochemical features of the hair follicle. In The Biology of Wool and Hair, Structure and Function of the Hair Follicle; Rogers, G.E., Reis, P.J., Ward, K.A., Marshall, R.C., Eds.; Springer Book Archive., Springer International Publishing AG: Berlin, Germany, 1989; pp. 69-85.

15. Swift, J.A. The electron histochemistry of cysteine-containing proteins in thin transverse sections of human hair. J. R. Microsc. Soc. 1968, 88, 449-460. [CrossRef] [PubMed]

16. Clément, J.L.; Le Pareux, A.; Ceccaldi, P.F. Contribution to hair medulla study (Author transl.). Ann. Dermatol. Venereol. 1981, 108, 849-857. [PubMed]

17. Matolsty, A.G. A study of the medullary cells of hair. Exp. Cell Res. 1953, 5, 98-110.

18. Langbein, L.; Yoshida, H.; Praetzel-Wunder, S.; Parry, D.A.; Schweizer, J. The keratins of the human beard hair medulla: The riddle in the middle. J. Investig. Dermatol. 2010, 130, 55-73. [CrossRef] [PubMed]

19. Logan, R.I.; Rivett, D.E.; Tucker, D.J.; Hudson, A.H. Analysis of the intercellular and membrane lipids of wool and other animal fibers. Text. Res. J. 1989, 59, 109-113. [CrossRef]

20. Mazukawa, Y.; Narita, H.; Imokawa, G. Characterization of the lipid composition at the proximal root regions of human hair. J. Cosmet. Chem. 2005, 56, 1-16. [CrossRef]

21. Wertz, P.W.; Downing, D.T. Integral lipids of mammalian hair. Comp. Biochem. Physiol. B Comp. Biochem. 1989, 92, 759-761. [CrossRef]

22. Weitkamp, A.W.; Smiljanic, A.M.; Rothman, S. The free fatty acids of human hair fat. J. Am. Chem. Soc. 1947, 69, 1936-1939. [CrossRef] [PubMed]

23. Wu, Y.; Chen, G.; Ji, C.; Hoptroff, M.; Jones, A.; Collins, L.Z.; Janssen, H.G. Gas chromatography-mass spectrometry and Raman imaging measurement of squalene content and distribution in human hair. Anal. Bioanal. Chem. 2016, 408, 2357-2362. [CrossRef] [PubMed]

24. Lee, W.S. Integral hair lipid in human hair follicle. J. Dermatol. Sci. 2011, 64, 153-158. [CrossRef] [PubMed]

25. Mahrle, G.; Orfanos, C.E. The spongious keratin and the medulla of human scalp hair. Arch. Derm. Res. 1971, 241, 305-316. [CrossRef]

26. Kreplak, L.; Briki, F.; Duvault, Y.; Doucet, J.; Merigoux, C.; Leroy, F.; Lévêque, J.L.; Miller, L.; Carr, G.L.; Williams, G.P.; et al. Profiling lipids across Caucasian and Afro-American hair transverse cuts, using synchrotron infrared microspectrometry. Int. J. Cosmet. Sci. 2001, 23, 369-374. [CrossRef] [PubMed]

27. Maeda, K. Analysis of ultraviolet radiation wavelengths causing hardening and reduced elasticity of collagen gels in vitro. Cosmetics 2018, 5, 14. [CrossRef]

28. Maeda, K.; Yamazaki, J.; Okita, N.; Shimotori, M.; Igarashi, K.; Sano, T. Mechanism of cuticle hole development in human hair due to UV-radiation exposure. Cosmetics 2018, 5, 24. [CrossRef] 
29. Maeda, D. Method for Producing Organic Compound Modified Inorganic Filler and Organic Compound Modified Inorganic Filler. Japan Patent Application No. WO2016166823A1, 31 April 2016.

30. Tipson, R.S. Functional groups in carbohydrates and their derivatives. In Infrared Spectrosc. Carbohydr-A Review of the Literature; Natl. Bur. Stand. Monogr. 110; U. S. Department of Commerce: Washington, DC, USA, 1968; pp. 5-8.

31. Amankwa, E. Raman and Surface-Enhanced Raman Spectroscopy of Fatty Acids and Lipids. 2016. Available online: http:/ / urn.fi/urn:nbn:fi:uef-20170094 (accessed on 17 March 2108).

32. Lee, D.H.; Condrate, R.A.; Lacourse, W.C. FTIR spectral characterization of thin film coatings of oleic acid on glasses. Part II Coatings on glass from different media such as water, alcohol, benzene and air. J. Mater. Sci. 2000, 35, 4961-4970. [CrossRef]

33. Picardo, M.; Ottaviani, M.; Camera, E.; Mastrofrancesco, A. Sebaceous gland lipids. Dermatoendocrinol. 2009, 1, 68-71. [CrossRef] [PubMed]

34. Morigaki, K.; Walde, P. Giant vesicle formation from oleic acid/sodium oleate on glass surfaces induced by adsorbed hydrocarbon molecules. Langmuir 2002, 18, 10509-10511. [CrossRef]

35. Fukuda, H.; Goto, A.; Yoshioka, H.; Goto, R.; Morigaki, K.; Walde, P. Electron spin resonance study of the $\mathrm{pH}$-induced transformation of micelles to vesicles in an aqueous oleic acid/oleate system. Langmuir 2001, 17, 4223-4231. [CrossRef]

36. Nawa, E.; Nishigaki, Y.; Yamamoto, D.; Shioi, A. Rhythmic shape change of a vesicle under a pH gradient. Soft Matter 2013, 9, 7832-7842. [CrossRef]

37. Takahashi, T.; Yoshida, S. Distribution of glycolipid and unsaturated fatty acids in human hair. Lipids 2014, 49, 905-917. [CrossRef] [PubMed]

(C) 2018 by the authors. Licensee MDPI, Basel, Switzerland. This article is an open access article distributed under the terms and conditions of the Creative Commons Attribution (CC BY) license (http:/ / creativecommons.org/licenses/by/4.0/). 